\title{
FRACTIONAL POWERS OF OPERATORS DEFINED ON A FRÉCHET SPACE
}

\author{
by W. LAMB \\ (Received 23rd September 1983)
}

\section{Introduction}

The problem of finding a suitable representation for a fractional power of an operator defined in a Banach space $X$ has, in recent years, attracted much attention. In particular, Balakrishnan [1], Hövel and Westphal [3] and Komatsu [4] have examined the problem of defining the fractional power $(-A)^{\alpha}$ for closed densely-defined operators $A$ such that

(i) $\lambda \in \rho(A)$ (the resolvent set of $A$ ) for each $\lambda>0$;

(ii) $\left\|\lambda(\lambda I-A)^{-1}\right\|<M<\infty$ for all $\lambda>0$.

Although the methods presented in [1], [3] and [4] differ to some extent, each is based primarily on the Bochner integral

$$
(-A)^{\alpha} f=-\pi^{-1} \sin (\pi \alpha) \int_{0}^{\infty} \lambda^{\alpha-1}(\lambda I-A)^{-1} A f d \lambda
$$

which, for $0<\operatorname{Re} \alpha<1$, defines $(-A)^{\alpha}$ as an operator on some suitably restricted subspace of $X$. In addition, the expected properties of fractional powers such as the index law

$$
(-A)^{\alpha}(-A)^{\beta}=(-A)^{\alpha+\beta} \quad(\operatorname{Re} \alpha, \operatorname{Re} \beta>0)
$$

are established in each case.

In this paper, we relax the condition that $X$ be a Banach space and consider the case where the operator $A$ is defined on a Fréchet space. By imposing conditions of a nature similar to (1.1), it will be shown that formula (1.2), interpreted slightly differently, can be used as the basis for defining fractional powers for $\operatorname{Re} \alpha>0$. Properties associated with these fractional powers, such as (1.3), will then be established in a natural manner by making certain modifications to the existing Banach space theory.

The motivation for developing a theory of fractional powers within this more general framework is the following.

Firstly, our main objective is to solve equations which can be expressed in the form

$$
(-A)^{\alpha} x=y \quad(\operatorname{Re} \alpha>0)
$$


where $x$ and $y$ are members of some function space $X$. It is our intention to study particular equations of this type in a future paper. As a first step, it is natural to attempt to extend the semi-group $\left\{(-A)^{\alpha} ; \operatorname{Re} \alpha>0\right\}$ to a group $\left\{\left(-A^{\alpha} ; \alpha \in \mathbb{C}\right\}\right.$ such that, for any complex numbers $\alpha$ and $\beta$, and $x \in X$,

(i) $(-A)^{\alpha}: X \rightarrow X$;

(ii) $(-A)^{\alpha}(-A)^{\beta} x=(-A)^{\alpha+\beta} x$;

(iii) $(-A)^{0} x=x$.

For such a group, the inverse of any operator $(-A)^{\alpha}$ is $(-A)^{-\alpha}$ so that the solution of (1.4) is given by $x=(-A)^{-\alpha} y$. Unfortunately, before this extension process can be carried out, certain additional constraints involving the existence of the inverse operator $A^{-1}$ have to be imposed. These have the undesirable effect of restricting the size of the function space $X$. One way of overcoming this difficulty is to utilise the theory of generalised functions. Equation (1.4) is then replaced by the weaker version

$$
(-\tilde{A})^{\alpha} f=g
$$

where $f$ and $g$ belong to some space of generalised functions $Y^{\prime}$ and $\tilde{A}$ denotes the extension of the operator $A$ to this space. If we adopt the definition given by Zemanian [12], then $Y^{\prime}$ is the dual of a space of testing functions $Y$ which is either a Fréchet space or the inductive limit of a family of such spaces and, in general, neither of these is a Banach space. Moreover, if, as frequently happens, the generalised operator $\tilde{A}$ is defined to be the adjoint of some operator $A^{*}$ (the formal adjoint of $A$ ) which acts upon $Y$, then we would expect $(-\tilde{A})^{\alpha}$ to be the adjoint of $\left(-A^{*}\right)^{\alpha}$, provided the latter exists. This indicates that, to make any progress in studying equation (1.6), a theory of fractional powers must first be developed for operators defined on a Fréchet space.

A second justification for this theory is in a similar vein. Recent developments in the subject of generalised functions have included the extension of certain operators of fractional calculus to various spaces of generalised functions. Prominent in this has been McBride [7] who has successfully tackled the problem of extending modifications of the Riemann-Liouville and Weyl operators of fractional integration. These operators behave, in essence, like fractional powers of the basic integral operators $J$ and $K$, defined on suitable functions $f$ by

$$
J f(x)=\int_{0}^{x} f(t) d t ; K f(x)=\int_{x}^{\infty} f(t) d t
$$

and therefore the work presented in this paper can be regarded as a natural progression from that carried out by McBride.

We begin in Section 2 by introducing some of the notation which is used throughout the text. For convenience, we also state some facts concerning the calculus of functions which assume values in an arbitrary Fréchet space $X$.

In Section 3 we examine operators which satisfy certain conditions of equi-continuity and establish various spectral results. These are used in Section 4 where a theory of fractional powers is developed for a class of operators $P(X)$. For $A \in P(X),(-A)^{\alpha}$ is defined by a formula based on (1.2) and various properties associated with fractional 
powers are established. In particular, it is shown that the family of operators $\left\{(-A)^{\alpha} ; \operatorname{Re} \alpha>0\right\}$ so defined forms a semigroup and, by imposing certain additional restrictions, we demonstrate that this can be extended to a group $\left\{(-A)^{\alpha} ; \alpha \in \mathbb{C}\right\}$ which satisfies (1.5). Throughout we concentrate only on continuous operators. This, however, causes no serious limitations since future applications lie in the field of generalised functions. There the standard practice is to construct a space of testing functions which ensures that the operator under investigation possesses properties such as continuity and invertibility.

In Section 5 we turn our attention to the dual space $X^{\prime}$ and show that a corresponding theory of fractional powers can be developed for operators defined on $X^{\prime}$. This is accomplished by employing some basic results on adjoint operators.

Finally, in Section 6, we indicate how this theory can readily be applied to certain classes of generalised functions.

For brevity, we present only the basic theory in this paper. In a future paper we hope to discuss examples involving certain classical and generalised integral and differential operators and consider applications to a class of hypergeometric integral equations.

\section{Notation and basic definitions}

Unless otherwise stated, $X$ will denote a Fréchet space equipped with the topology generated by a separating family of seminorms $S=\left\{\gamma_{k}\right\}_{k=0}^{\infty}$. To represent the dual space of $X$, we use the customary notation of $X^{\prime}$, with the value assigned to $x \in X$ by $f \in X^{\prime}$ expressed as $(f, x)$. Similarly, we denote the class of all continuous linear operators mapping $X$ into itself by $L(X)$ and use the symbol $L_{s}(X)$ for the same set equipped with the topology of pointwise convergence (see [11, p. 110]). This topology may be regarded as that generated by the (generally) uncountable collection of seminorms $\left\{\tau_{k, x} ; x \in X\right.$, $k=0,1, \ldots\}$ where

$$
\tau_{k, x}(A)=\gamma_{k}(A x) .
$$

Since $X$ is a Fréchet space, it follows from [9, Theorem 2.8] that $L_{s}(X)$ is complete.

In developing the theory, we shall frequently use results from the calculus of vectorvalued functions. Since the concepts of strong (weak) continuity and holomorphy are well-known [9, p. 78], we pause here only to discuss how the Riemann integral of a function assuming values in a Fréchet space $X$ may be defined. If $\phi$ maps a compact interval $[a, b]$ into $X$, then we define the Riemann integral of $\phi$ over $[a, b]$ in the obvious manner by using the seminorms $\gamma_{k} \in S$ instead of the Euclidean norm in the definition of the Riemann integral of a numerically-valued function. When the Riemann integral of $\phi$ over $[a, b]$ exists, we write it as $\int_{a}^{b} \phi(\lambda) d \lambda$.

Since $X$ is a Fréchet space, it is clear that whenever the Riemann integral exists in $X$, it is necessarily unique. The question of existence is answered by the following lemma.

Lemma 2.1. If $\phi:[a, b] \rightarrow X$ is a strongly continuous vector-valued function, then the Riemann integral $\int_{a}^{b} \phi(\lambda) d \lambda$ exists in $X$. Moreover,

$$
\gamma_{k}\left(\int_{a}^{b} \phi(\lambda) d \lambda\right) \leqq \int_{a}^{b} \gamma_{k}(\phi(\lambda)) d \lambda
$$

for any $\gamma_{k} \in S$. 
Proof. A proof of this lemma can be found in [5, pp. 26-27].

\section{Spectral theory}

The spectrum and resolvent set of an operator $A \in L(X)$ are defined in the normal fashion. Thus a complex number $\lambda$ belongs to the resolvent set $\rho(A)$ if the resolvent operator $R(\lambda ; A) \equiv(\lambda I-A)^{-1}$ exists as an operator in $L(X)$. The complement of $\rho(A)$ in $\mathbb{C}$ is the spectrum of $A$, denoted by $\sigma(A)$.

To enable us to deduce certain facts concerning the operators $R(\lambda ; A), \lambda \in \rho(A)$, we restrict our attention to a subset $\rho^{*}(A)$ of $\rho(A)$. This subset is defined in the following manner.

Definition 3.1. A complex number $\lambda$ belongs to the set $\rho^{*}(A)$ if

(i) $\lambda \in \rho(A)$

(ii) for each seminorm $\gamma_{k} \in S$, there exists a seminorm $\gamma_{l} \in S$ such that

$$
\gamma_{k}\left(\left[C_{\lambda} R(\lambda ; A)\right]^{n} x\right) \leqq \gamma_{l}(x)
$$

for all $n=1,2, \ldots$ and $x \in X$, where $C_{\lambda}$ is a positive constant depending only upon $\lambda$.

Remark 3.2. It can easily be verified that, as a result of (3.1), the family of operators $\left\{\left[C_{\lambda} R(\lambda ; A)\right]^{n}\right\}_{n=1}^{\infty}$ is equi-continuous in $n$ (see $[9$, p. 43]).

Obviously the constant $C_{\lambda}$ in (3.1) may be replaced by any other positive constant $D_{\lambda}$ such that $D_{\lambda}<C_{\lambda}$. Therefore we can define

$$
C_{\lambda}(A)=\sup \left\{C_{\lambda} \text { :inequality (3.1) is valid }\right\}
$$

It is interesting to note that, when $X$ is a Banach space,

$$
\rho^{*}(A)=\rho(A) \quad \text { and } \quad C_{\lambda}(A)=\|R(\lambda ; A)\|_{L(X)}^{-1} .
$$

Theorem 3.3. Let $A \in L(X)$ be such that $\rho^{*}(A) \neq \varnothing$. Then

(i) $\rho^{*}(A)$ is open;

(ii) $R(\lambda ; A)$ is a strongly holomorphic $L_{s}(X)$-valued function of $\lambda$ on $\rho^{*}(A)$.

Proof. (i) To prove that $\rho^{*}(A)$ is open, we merely remark that if $\xi \in \rho^{*}(A)$ and $\mu$ is such that $|\mu-\xi|<C_{\xi}<C_{\xi}(A)$, then the series $\sum_{m=0}^{\infty}[R(\xi ; A)]^{m+1}(\xi-\mu)^{m}$ converges to $R(\mu ; A)$ in $L_{s}(X)$. Moreover, for any $\gamma_{k} \in S$, there exists a $\gamma_{l} \in S$ such that

$$
\gamma_{k}\left([R(\mu ; A)]^{n} x\right) \leqq\left(C_{\mu}\right)^{n} \gamma_{l}(x) \quad(x \in X)
$$

where $C_{\mu}=\left(C_{\xi}-|\mu-\xi|\right)^{-1}$. Thus $\mu \in \rho^{*}(A)$ and it follows that $\rho^{*}(A)$ is open.

(ii) We first verify that $R(\lambda ; A)$ is continuous on any compact subset $K$ of $\rho^{*}(A)$. To this end, let $\lambda, \mu \in K$ with $\lambda$ fixed. 
Then

$$
\begin{aligned}
& \tau_{k, x}(R(\lambda ; A)-R(\mu ; A)) \\
& \quad=\gamma_{k}(R(\lambda ; A) x-R(\mu ; A) x) \quad(\text { from }(2.1)) \\
& \left.\quad \leqq|\mu-\lambda| M_{1}(k) \max _{1 \leqq i \leqq n}\left\{\gamma_{k_{i}}(R(\mu ; A) x)\right\} \quad \text { (for some indices } k_{1}, k_{2}, \ldots, k_{n}\right)
\end{aligned}
$$

where $M_{1}(k)$ is a constant depending on $k$ and (3.2) has been obtained by utilising [12, Lemma 1.10-1] in conjunction with the resolvent equation. Now let $\xi$ be fixed in $K$, let $x$ be an arbitrary element in $X$ and let $0<C_{\xi}<C_{\xi}(A)$. Then, for any $\mu \in S\left(\xi, C_{\xi} / 2\right)$, where

$$
S\left(\xi, C_{\xi} / 2\right)=\left\{z:|z-\xi|<C_{\xi} / 2\right\}
$$

we can express $R(\mu ; A) x$ as

$$
R(\mu ; A) x=(\xi-\mu)^{-1} \sum_{n=0}^{\infty}([\xi-\mu] R(\xi ; A))^{n+1} x
$$

Moreover, by Definition 3.1, for any $\gamma_{k} \in S$ there exists a $\gamma_{l(\xi)} \in S$ such that

$$
\gamma_{k}\left(\left[C_{\xi} R(\xi ; A)\right]^{n} x\right) \leqq \gamma_{l(\xi)}(x) \quad(n=1,2, \ldots) .
$$

Hence, from (3.3),

$$
\gamma_{k}(R(\mu ; A) x) \leqq\left(2 / C_{\xi}\right) \gamma_{l(\xi)}(x)
$$

Since $K$ is compact, there exist a finite sequence $\left\{\xi_{j}\right\}_{j=1}^{m}$ and constants $C_{\xi_{j}}<C_{\xi_{j}}(A)$ $(j=1,2, \ldots, m)$ such that $K \subset \bigcup_{j=1}^{m} S\left(\xi_{j}, C_{\xi_{j}} / 2\right)$. If we set $M_{2}=\max _{1 \leqq j \leqq m}\left\{2 / C_{\xi_{j}}\right\}$, then we can deduce that

$$
\gamma_{k}(R(\mu ; A) x) \leqq M_{2} \max _{1 \leqq j \leqq m}\left\{\gamma_{l\left(\xi_{j}\right)}(x)\right\}
$$

for any $\mu \in K$. Thus, from (3.2) and (3.4),

$$
\begin{gathered}
\tau_{k, x}(R(\lambda ; A)-R(\mu ; A)) \\
\leqq|\mu-\lambda| M_{1}(k) M_{2} \max _{i, j}\left\{\gamma_{l_{i}\left(\xi_{j}\right)}(x)\right\} \rightarrow 0
\end{gathered}
$$

as $\mu \rightarrow \lambda$ in $K$. Since $\gamma_{k}$ was arbitrarily chosen, it follows that $R(\lambda ; A)$ is continuous on $K$. The result can now be deduced by making minor modifications to the argument used for the case when $X$ is a Banach space [8, p. 40].

\section{Fractional powers of operators defined on Fréchet spaces}

In this section we shall be concerned with the following class of operators. 
Definition 4.1. The set $P(X)$ consists of continuous linear operators $A: X \rightarrow X$ which are such that

(i) $R(\lambda ; A) \in L(X)$, for all $\lambda>0$;

(ii) for each $\gamma_{k} \in S$ there exists a $\gamma_{l} \in S$ such that

$$
\gamma_{k}\left([C \lambda R(\lambda ; A)]^{n} x\right) \leqq \gamma_{l}(x)
$$

for all $x \in X, \lambda>0$ and $n=1,2, \ldots$, where $C$ is a positive constant independent of $\lambda$, $n$ and $x$.

The following comments are appropriate. Firstly, condition (4.2) can be used to establish that the family of operators $\left\{[C \lambda R(\lambda ; A)]^{n}\right\}$ is equi-continuous in $\lambda>0$ and $n=1,2, \ldots$. Secondly, when $X$ is a Banach space, (4.1) and (4.2) reduce to the conditions stated in (1.1) and therefore results concerning fractional powers of continuous operators defined on a Banach space can be deduced as a special case of the theory developed here.

Definition 4.2. Let $A \in P(X)$ and let $\alpha \in \mathbb{C}$ with $\operatorname{Re} \alpha>0$. Then we define the fractional power $(-A)^{\alpha}$ by

$$
\begin{aligned}
(-A)^{\alpha} x= & -\pi^{-1} \sin (\pi \alpha) \int_{0}^{\infty} \lambda^{\alpha-1}\left[R(\lambda ; A)-\lambda /\left(1+\lambda^{2}\right)\right] A x d \lambda \\
& -\sin (\pi a / 2) A x \quad(0<\operatorname{Re} \alpha<2)
\end{aligned}
$$

and

$$
(-A)^{\alpha} x=(-A)^{\alpha-n}(-A)^{n} x \quad(n<\operatorname{Re} \alpha<n+2, n=1,2, \ldots)
$$

where $x \in X$ and the integral in (4.3) is regarded as a strong improper Riemann integral in $X$ of the form

$$
\lim _{\substack{M \rightarrow \infty \\ \varepsilon \rightarrow 0+}} \int_{\varepsilon}^{M} \lambda^{\alpha-1}\left[R(\lambda ; A)-\lambda /\left(1+\lambda^{2}\right)\right] A x d \lambda
$$

We remark that here, and in the sequel, any expression of the form $\lambda^{\alpha}$, where $\lambda>0$ and $\alpha \in \mathbb{C}$, is interpreted as $\exp (\alpha \log (\lambda))$ where $\log (\lambda)$ assumes its principal branch. We also note that Definition 4.2 can give rise to two representations for the fractional power $(-A)^{\alpha}$. For example, suppose $\operatorname{Re} \alpha \in(n, n+1), n>1$. If we regard this interval as a subset of $(n, n+2)$ then $(-A)^{\alpha}=(-A)^{\alpha-n}(-A)^{n}$. Alternatively, if we interpret it as a subset of $(n-1, n+1)$ then $(-A)^{\alpha}=(-A)^{\alpha-n+1}(-A)^{n-1}$. We shall show later that this does not lead to any inconsistencies.

Theorem 4.3. If $A \in P(X)$, then $\rho^{*}(A)$ is non-empty and open. Moreover,

(i) $R(\lambda ; A)$ is a strongly holomorphic $L_{s}(X)$-valued function of $\lambda$ on $\rho^{*}(A)$;

(ii) for any fixed $x \in X, R(\lambda ; A) x$ is a strongly holomorphic $X$-valued function of $\lambda$ on $\rho^{*}(A)$. 
Proof. Since $(0, \infty) \subset \rho^{*}(A)$, it is clear that $\rho^{*}(A)$ is non-empty. The remainder of the theorem can be deduced from Theorem 3.3.

Theorem 4.4. If $A \in P(X)$, then $(-A)^{\alpha}$, defined by (4.3) and (4.4), is a continuous linear operator on $X$.

Proof. Let $0<\operatorname{Re} \alpha<2$ and consider the improper integral (4.5). By Lemma 2.1 and Theorem 4.3(ii), for any fixed $x \in X$, each integral

$$
\int_{\varepsilon}^{M} \lambda^{\alpha-1}\left[R(\lambda ; A)-\lambda /\left(1+\lambda^{2}\right)\right] A x d \lambda
$$

exists in $X$ as a strong Riemann integral. Moreover, if $\gamma_{k} \in S$, then

$$
\begin{aligned}
& \gamma_{k}\left(\int_{\varepsilon}^{M} \lambda^{\alpha-1}\left[R(\lambda ; A)-\lambda /\left(1+\lambda^{2}\right)\right] A x d \lambda\right) \\
& \leqq \int_{\varepsilon}^{M} \lambda^{\operatorname{Re} \alpha-1} \gamma_{k}\left(\left[R(\lambda ; A)-\lambda /\left(1+\lambda^{2}\right)\right] A x\right) d \lambda \quad \text { (by Lemma 2.1) } \\
& \leqq \int_{\varepsilon}^{M}\left[\gamma_{k}(\lambda R(\lambda ; A) x)+\gamma_{k}(x)+\gamma_{k}\left(\lambda R(\lambda ; A) A^{2} x\right)\right] \lambda^{\operatorname{Re} \alpha-1} /\left(1+\lambda^{2}\right) d \lambda
\end{aligned}
$$

Now, from (4.2) and the continuity of $A^{2}$ on $X$, we can find seminorms $\gamma_{l}$ and $\left\{\gamma_{k(i)}\right\}_{i=1}^{m}$ in $S$ and constants $C>0$ and $N(k)>0$ (independent of $x$ ) such that the expression in (4.6) is bounded above by

$$
\left[\left(\gamma_{l}(x)+N(k) \max _{i}\left\{\gamma_{k(i)}(x)\right\}\right) / C+\gamma_{k}(x)\right] \int_{\varepsilon}^{M} \lambda^{\mathrm{Re} \alpha-1} /\left(1+\lambda^{2}\right) d \lambda
$$

By letting $\varepsilon \rightarrow 0+$ and $M \rightarrow \infty$, we see that the integral in (4.7) converges to $(\pi / 2) \operatorname{cosec}(\pi \operatorname{Re} \alpha / 2)$. It follows from this in a routine manner that $(-A)^{\alpha}$ is a continuous linear operator on $X$ for $\operatorname{Re} \alpha>0$.

Theorem 4.5. If $A \in P(X)$ and $\operatorname{Re} \alpha \in(n-1, n) \quad(n=1,2, \ldots)$, then $(-A)^{\alpha}$ has the equivalent representation

$$
(-A)^{\alpha} x=\pi^{-1} \sin (\pi[\alpha+1-n]) \int_{0}^{\infty} \lambda^{\alpha-n} R(\lambda ; A)(-A)^{n} x d \lambda
$$

Proof. Suppose that $0<\operatorname{Re} \alpha<1$. Then, from (4.3),

$$
\begin{aligned}
(-A)^{\alpha} x= & \pi^{-1} \sin (\pi \alpha) \int_{0}^{\infty} \lambda^{\alpha-1} R(\lambda ; A)(-A x) d \lambda \\
& +(A x) \pi^{-1} \sin (\pi \alpha) \int_{0}^{\infty} \lambda^{\alpha} /\left(1+\lambda^{2}\right) d \lambda-(A x) \sin (\pi \alpha / 2)
\end{aligned}
$$


and this reduces to (4.8) with $n=1$ when the integral $\int_{0}^{\infty} \lambda^{\alpha} /\left(1+\lambda^{2}\right) d \lambda$ is evaluated as $(\pi / 2) \operatorname{cosec}([1+\alpha] \pi / 2)$. Similarly, if we use the identity

$$
\lambda R(\lambda ; A)(-A x)=-A x-R(\lambda ; A)(-A)^{2} x
$$

then the result can also be verified for $\operatorname{Re} \alpha \in(1,2)$. The proof now follows from (4.4).

If we now return to the question of the consistency of the definition given in (4.3) and (4.4), then we can conclude that for $\operatorname{Re} \alpha \in(n, n+1)$

$$
(-A)^{\alpha} x=\pi^{-1} \sin (\pi[\alpha-n]) \int_{0}^{\infty} \lambda^{\alpha-n-1} R(\lambda ; A)(-A)^{n+1} x d \lambda
$$

regardless of whether $(n, n+1)$ is interpreted as a subset of $(n-1, n+1)$ or as a subset of $(n, n+2)$.

We shall now prove certain results concerning the operators $(-A)^{\alpha}$ which demonstrate that they possess the fundamental properties associated with fractional powers.

Theorem 4.6. If $A \in P(X)$, then $(-A)^{\alpha}$, defined by (4.3) and (4.4), is a strongly holomorphic $L_{s}(X)$-valued function of $\alpha$ in the region $\operatorname{Re} \alpha>0$.

Proof. We shall show that $(-A)^{\alpha} x$ is a strongly holomorphic $X$-valued function in the region $\operatorname{Re} \alpha>0$ for each fixed $x \in X$; see (2.1). Let $x$ be a fixed element in $X$ and let $0<\operatorname{Re} \alpha<2$. Then, by (4.3),

$$
\begin{aligned}
(-A)^{\alpha} x= & \pi^{-1} \sin (\pi \alpha) \int_{0}^{\infty} \lambda^{\alpha-1}\left[R(\lambda ; A)-\lambda /\left(1+\lambda^{2}\right)\right](-A x) d \lambda \\
& -(A x) \sin (\pi \alpha / 2) .
\end{aligned}
$$

Since $\sin (\pi \alpha)$ is an entire function of $\alpha$, it will suffice to verify that

$$
T(\alpha) x=\int_{0}^{\infty} \lambda^{\alpha-1}\left[R(\lambda ; A)-\lambda /\left(1+\lambda^{2}\right)\right](-A x) d \lambda
$$

is strongly holomorphic in $X$ with respect to $\alpha$ for $\operatorname{Re} \alpha>0$. We define

$$
T^{\prime}(\alpha) x=\int_{0}^{\infty} \lambda^{\alpha-1} \log (\lambda)\left[R(\lambda ; A)-\lambda /\left(1+\lambda^{2}\right)\right](-A x) d \lambda(\operatorname{Re} \alpha>0)
$$

where (4.10) is obtained from (4.9) by differentiating formally under the integral sign with respect to $\alpha$. We shall demonstrate that, for each $\gamma_{k} \in S$ and for each $\alpha$ in the halfplane $\operatorname{Re} \alpha>0$,

$$
\gamma_{k}\left([T(\alpha+h) x-T(\alpha) x] / h-T^{\prime}(\alpha) x\right) \rightarrow 0
$$


as the complex increment $h \rightarrow 0$. To this end, consider

$$
\begin{gathered}
\gamma_{k}\left([T(\alpha+h) x-T(\alpha) x] / h-T^{\prime}(\alpha) x\right) \\
\leqq \sup _{0 \leqq \lambda \leqq 1}\left|f_{h}(\lambda)\right| \int_{0}^{\infty} \lambda^{\operatorname{Re} \alpha-\eta-1} \gamma_{k}\left(\left[R(\lambda ; A)-\lambda /\left(1+\lambda^{2}\right)\right](-A x)\right) d \lambda \\
+\sup _{1 \leqq \lambda<\infty}\left|g_{h}(\lambda)\right| \int_{0}^{\infty} \lambda^{\text {Re } \alpha+\varepsilon-1} \gamma_{k}\left(\left[R(\lambda ; A)-\lambda /\left(1+\lambda^{2}\right)\right](-A x) d \lambda\right.
\end{gathered}
$$

where $\eta$ and $\varepsilon$ are such that $\operatorname{Re} \alpha-\eta \in(0,2)$ and $\operatorname{Re} \alpha+\varepsilon \in(0,2)$

and

$$
\begin{gathered}
f_{h}(\lambda)=\lambda^{\eta}\left(\left[\lambda^{h}-1\right] / h-\log (\lambda)\right) \\
g_{h}(\lambda)=\lambda^{-\varepsilon}\left(\left[\lambda^{h}-1\right] / h-\log (\lambda)\right) .
\end{gathered}
$$

It can be shown that, as $h \rightarrow 0$ in any manner, $\sup _{0 \leqq \lambda \leqq 1}\left|f_{h}(\lambda)\right|$ and $\sup _{1 \leqq \lambda<\infty}\left|g_{h}(\lambda)\right|$ both converge to $0[6$, p. 37]. Moreover, by an argument similar to that used in the proof of Theorem 4.4, it can be verified that the integrals appearing in (4.11) are both finite. This establishes that $(-A)^{x} x$ is strongly holomorphic in $X$ for $\operatorname{Re} \alpha \in(0,2)$ and the general result follows directly from (4.4).

Theorem 4.7. Let $x \in X$ be such that $\gamma_{k}(\lambda R(\lambda ; A) x) \rightarrow 0$ as $\lambda \rightarrow 0+$ for all $\gamma_{k} \in S$. Then, if $A \in P(X),(-A)^{\alpha} x$ converges strongly to $x$ as $\alpha \rightarrow 0$ in any fixed sector about 0 of the form $\{\alpha:|\arg \alpha|<\pi / 2-\eta, 0<\eta<\pi / 2\}$.

Proof. Since the proof is similar to that presented in $[11$, p. 266] for the case when $X$ is a Banach space, details are omitted.

Theorem 4.8. If $\alpha=n$, where $n$ is a positive integer, then $(-A)^{\alpha}$ is equal to the $n$th iterate of $(-A)$.

Proof. In view of (4.4), it is sufficient to prove this for the case $\alpha=1$. From (4.3)

$$
\begin{aligned}
(-A)^{1} x= & \pi^{-1} \sin (\pi) \int_{0}^{\infty}\left[R(\lambda ; A)-\lambda /\left(1+\lambda^{2}\right)\right](-A x) d \lambda \\
& +\sin (\pi / 2)(-A x) \\
= & -A x
\end{aligned}
$$

since the integral exists in $X$ as a strong improper Riemann integral. 
We now verify that, under suitable conditions, the fractional powers $(-A)^{\alpha}$ satisfy the two index laws $(-A)^{\alpha}(-A)^{\beta}=(-A)^{\alpha+\beta}$ and $\left[(-A)^{\alpha}\right]^{\beta}=(-A)^{\alpha \beta}$. First we require the following lemma.

Lemma 4.9. If $A \in P(X), 0<\alpha<1$ and $\lambda>0$, then the resolvent operator $R\left(\lambda ;-(-A)^{\alpha}\right)$ exists in $L_{s}(X)$ and is given by

$$
R\left(\lambda ;-(-A)^{\alpha}\right) x=\int_{0}^{\infty} g_{\lambda, a}(u) R(u ; A) x d u \quad(x \in X),
$$

where $g_{\lambda, \alpha}$ is defined on $[0, \infty)$ by

$$
g_{\lambda, \alpha}(u)=\pi^{-1} \sin (\pi \alpha) u^{\alpha}\left[\lambda^{2}+2 \lambda u^{\alpha} \cos (\pi \alpha)+u^{2 \alpha}\right]^{-1} .
$$

Proof. If we remark that $g_{\lambda, \alpha}$ possesses the properties

(i) $g_{\lambda, \alpha}(u) \geqq 0$ for all $u \geqq 0$,

(ii) $\int_{0}^{\infty} g_{\lambda, a}(u) u^{-1} d u=\lambda^{-1}$ for $\lambda>0$ and

(iii) $\int_{0}^{\infty} g_{\lambda, a}(u)(s+u)^{-1} d u=\left(\lambda+s^{\alpha}\right)^{-1}[3$, p. 191], then it follows that (4.12), interpreted as an improper Riemann integral, defines an operator in $L_{s}(X)$. Moreover, for $x \in X$,

$$
\begin{gathered}
(-A)^{\alpha} \int_{0}^{\infty} g_{\lambda, \alpha}(u) R(u ; A) x d u \\
=-\pi^{-1} \sin (\pi \alpha) \int_{0}^{\infty} \int_{0}^{\infty} t^{\alpha-1} g_{\lambda, \alpha}(u) R(t ; A) R(u ; A) A x d u d t \\
=-\pi^{-1} \sin (\pi \alpha) \int_{0}^{\infty} t^{\alpha-1} R(t ; A) A x d t \int_{0}^{\infty} g_{\lambda, \alpha}(u)(u-t)^{-1} d u \\
-\pi^{-1} \sin (\pi \alpha) \int_{0}^{\infty} g_{\lambda, \alpha}(u) R(u ; A) A x d u \int_{0}^{\infty} t^{\alpha-1}(t-u)^{-1} d t,
\end{gathered}
$$

where the resolvent equation has been used in conjunction with an interchange in the order of integration to obtain (4.14). The interchange may be justified by an application of the Fubini-Tonelli theorem [5, pp. 128-131]. If we now use the formulae given in [2, p. 240] relating the Hilbert transform to the Stieltjes transform, we can evaluate the inner integrals in (4.14) obtaining

$$
\begin{aligned}
& -\pi^{-1} \sin (\pi \alpha) \int_{0}^{\infty} \mu^{\alpha-1} R(\mu ; A) A x\left[\frac{\lambda+\mu^{\alpha} \cos (\pi \alpha)-\mu^{\alpha} \cos (\pi \alpha)}{\lambda^{2}+2 \lambda \mu^{\alpha} \cos (\pi \alpha)+\mu^{2 \alpha}}\right] d \mu \\
& =\int_{0}^{\infty} \lambda \mu^{-1} g_{\lambda, \alpha}(\mu)[I-\mu R(\mu ; A)] x d \mu \\
& =I x-\lambda \int_{0}^{\infty} g_{\lambda, \alpha}(\mu) R(\mu ; A) x d \mu .
\end{aligned}
$$


Hence

$$
\left(\lambda I+(-A)^{\alpha}\right) \int_{0}^{x} g_{\lambda, \alpha}(u) R(u ; A) x d u=x
$$

for all $x \in X$. Similarly, since the order of integration in (4.13) may be interchanged,

$$
\int_{0}^{\infty} g_{\lambda, \alpha}(u) R(u ; A)\left(\lambda I+(-A)^{\alpha}\right) x d u=x \quad(x \in X) .
$$

This completes the proof.

Theorem. 4.10. If $A \in P(X), x \in X$ and $\operatorname{Re} \beta>0$, then

(i) $(-A)^{\alpha}(-A)^{\beta} x=(-A)^{\alpha+\beta} x$, for $\operatorname{Re} \alpha>0$;

(ii) $\left[(-A)^{\alpha}\right]^{\beta} x=(-A)^{\alpha \beta} x$, for $0<\alpha<1$.

Proof. (i) This can be proved in the manner described in [11, p. 267]. (ii) First we establish that $-(-A)^{\alpha} \in P(X)$ for any $\alpha \in(0,1)$. Let $\gamma_{k} \in S, \lambda>0$ and $0<\alpha<1$. Then, from Lemmas 4.9 and 2.1 , for $x \in X$ and $n=1,2, \ldots$,

$$
\begin{gathered}
\gamma_{k}\left(\lambda^{n}\left[R\left(\lambda_{i} ;-(-A)^{\alpha}\right]^{n} x\right)\right. \\
\leqq \lambda^{n} \int_{0}^{\infty} g_{\lambda, \alpha}\left(u_{1}\right) \ldots \int_{0}^{\infty} g_{\lambda, \alpha}\left(u_{n}\right) \gamma_{k}\left(R\left(u_{1} ; A\right) \ldots R\left(u_{n} ; A\right) x\right) d u_{n} \ldots d u_{1} \\
\leqq \gamma_{l}(x)(\lambda / C)^{n} \int_{0}^{\infty} g_{\lambda_{,} \alpha}\left(u_{1}\right) u_{1}^{-1} d u_{1} \ldots \int_{0}^{\infty} g_{\lambda_{,} \alpha}\left(u_{n}\right) u_{n}^{-1} d u_{n} \\
=C^{-n} \gamma_{l}(x) .
\end{gathered}
$$

Hence $-(-A)^{\alpha} \in P(X)$ and therefore, by Theorem 4.4, $\left[(-A)^{\alpha}\right]^{\beta}$ exists as an operator in $L_{s}(X)$ provided that $\operatorname{Re} \beta>0$. The result now follows on using a proof identical to that given by Watanabe [10].

An inspection of Theorem 4.7 and part (i) of the previous theorem reveals that the operators $\left\{(-A)^{\alpha} ; \operatorname{Re} \alpha>0\right\}$, defined by (4.3) and (4.4), satisfy the semi-group property $(-A)^{\alpha}(-A)^{\beta}=(-A)^{\alpha+\beta}$ for $\operatorname{Re} \alpha, \operatorname{Re} \beta>0$, and also converge in a certain sense to the identity operator $I$ as $\alpha \rightarrow 0$. For the reasons given in Section 1, we would like to extend this semi-group to a group of operators $\left\{(-A)^{\alpha} ; \alpha \in \mathbb{C}\right\}$ satisfying (1.5). This extension is carried out in the following manner.

Let $A \in P(X)$ possess an inverse $A^{-1} \in L(X)$. Then, for $\operatorname{Re} \alpha>0$, the fractional power $(-A)^{\alpha}$ may be expressed as

$$
(-A)^{\alpha}=(-A)^{\alpha+1}(-A)^{-1}
$$

Now, from Theorem 4.6 and the fact that $(-A)^{-1} \in L(X)$, the right-hand side of $(4.15)$ is meaningful and strongly holomorphic in $L_{s}(X)$ for $\operatorname{Re} \alpha>-1$. Therefore we can use 
(4.15) to continue the definition of $(-A)^{\alpha}$ analytically to all values of $\alpha$ in the half-plane $\operatorname{Re} \alpha>-1$ and, by repeated application, to all complex values of $\alpha$. Thus we may define $(-A)^{\alpha}$, for any complex value of $\alpha$, by

$$
(-A)^{\alpha} x=(-A)^{\alpha+n}\left[(-A)^{-1}\right]^{n} x=\left[(-A)^{-1}\right]^{n}(-A)^{\alpha+n} x \quad(x \in X),
$$

provided only that the non-negative integer $n$ is such that $\operatorname{Re} \alpha+n>0$. It is a routine matter to verify that (4.16) is independent of the integer $n$ satisfying $\operatorname{Re} \alpha+n>0$.

We can immediately state the following facts.

Theorem 4.11. Let $A \in P(X)$ possess an inverse $A^{-1} \in L(X)$ and let $(-A)^{\alpha}$ be defined by (4.3) and (4.4) for $\operatorname{Re} \alpha>0$ and extended via (4.16) to all complex values of $\alpha$. Then

(i) $(-A)^{\alpha}$ is a homeomorphism of $X$ onto $X$ and its inverse is given by $\left[(-A)^{\alpha}\right]^{-1}=$ $(-A)^{-\alpha}$

(ii) $(-A)^{\alpha}$ is a strongly holomorphic $L_{s}(X)$-valued function of $\alpha$ in $\mathbb{C}$;

(iii) $(-A)^{\alpha}(-A)^{\beta}=(-A)^{\alpha+\beta}(\alpha, \beta \in \mathbb{C})$;

(iv) $(-A)^{\alpha}=(-A)^{n}$, the nth iterate of $-A$ when $\alpha$ is a positive integer $n ;(-A)^{\alpha}=$ $(-A)^{-n}$, the nth iterate of $-A^{-1}$ when $\alpha$ is a negative integer $-n$;

(v) $\left[(-A)^{\alpha}\right]^{\beta}=(-A)^{\alpha \beta}(0<\alpha<1, \beta \in \mathbb{C})$.

Proof. These results can be obtained in a straightforward manner by using (4.16) in conjunction with Theorems $4.6,4.8$ and 4.10 .

An interesting point emerges at this stage. From (4.17), it is seen that the inverse of $(-A)^{\alpha}$ is $(-A)^{-\alpha}$. When $\alpha$ is an integer $n,(-A)^{-n}=\left(-A^{-1}\right)^{n}$ so that $\left[(-A)^{n}\right]^{-1}=$ $\left(-A^{-1}\right)^{n}$. This raises the obvious question of whether or not the same result holds for non-integral values of $\alpha$. We answer this question in the following theorem.

Theorem 4.12. If $A \in P(X)$ has an inverse $A^{-1} \in L(X)$, then $A^{-1} \in P(X)$. Moreover, $(-A)^{-\alpha}=\left(-A^{-1}\right)^{\alpha}$.

Proof. From the identify

$$
\left(\lambda I-A^{-1}\right)^{-1}=-\lambda^{-1}\left(\lambda^{-1} I-A\right)^{-1} A \quad(\lambda>0),
$$

we can immediately deduce that $[0, \infty) \subset \rho\left(A^{-1}\right)$. Moreover, for $x \in X, \gamma_{k} \in S$ and $\lambda>0$,

$$
\begin{aligned}
\gamma_{k}( & {\left.\left[\lambda R\left(\lambda ; A^{-1}\right)\right]^{n} x\right) } \\
= & \gamma_{k}\left(\left[R\left(\lambda^{-1} ; A\right) A\right]^{n} x\right) \\
= & \gamma_{k}\left(\left[\lambda^{-1} R\left(\lambda^{-1} ; A\right)-I\right]^{n} x\right) \\
\quad & \leqq \sum_{j=0}^{n}\left(\begin{array}{l}
n \\
j
\end{array}\right) \gamma_{k}\left(\left[R\left(\lambda^{-1} ; A\right) \lambda^{-1}\right]^{j}[-I]^{n-j} x\right)
\end{aligned}
$$




$$
\begin{aligned}
& \leqq \gamma_{l}(x) \sum_{j=0}^{n}\left(\begin{array}{l}
n \\
j
\end{array}\right) C^{-j} \\
= & \left(1+C^{-1}\right)^{n} \gamma_{l}(x)
\end{aligned}
$$

and therefore condition (4.2) is also satisfied with $A$ replaced by $A^{-1}$. Hence $A^{-1} \in P(X)$. Now let $\operatorname{Re} \alpha \in(0,1)$. Then

$$
\begin{aligned}
\left(-A^{-1}\right)^{\alpha} x & =-\pi^{-1} \sin (\pi \alpha) \int_{0}^{\infty} \lambda^{\alpha-1} R\left(\lambda ; A^{-1}\right) A^{-1} x d \lambda \quad \text { (by (4.8)) } \\
& =\pi^{-1} \sin (\pi \alpha) \int_{0}^{\infty} \lambda^{\alpha-2} R\left(\lambda^{-1} ; A\right) x d \lambda \quad \text { (by (4.18)) } \\
& =\pi^{-1} \sin (\pi \alpha) \int_{0}^{\infty} u^{-\alpha} R(u ; A) x d u \\
& =(-A)^{1-\alpha}(-A)^{-1} x=(-A)^{-\alpha} x \quad \text { (by (4.8) and (4.16)) }
\end{aligned}
$$

Hence $(-A)^{-\alpha}=\left(-A^{-1}\right)^{\alpha}$ for $0<\operatorname{Re} \alpha<1$ and, since $(-A)^{-\alpha}$ and $\left(-A^{-1}\right)^{\alpha}$ are both entire functions of $\alpha$ (Theorem 4.11(ii)), it follows that $(-A)^{-\alpha}=\left(-A^{-1}\right)^{\alpha}$ for all complex values of $\alpha$. This completes the proof.

As a consequence of this theorem, if $A \in P(X)$ and $A^{-1}$ exists in $L(X)$, then all the results stated in Theorem 4.11 are valid when $A$ is replaced by $A^{-1}$. Furthermore, it is easily shown that Theorem $4.11(\mathrm{v})$ extends to

$$
\left[(-A)^{\alpha}\right]^{\beta}=(-A)^{\alpha \beta} \quad(-1<\alpha<1, \beta \in \mathbb{C}) .
$$

\section{Fractional powers of operators defined on $X^{\prime}$}

Having discussed fractional powers of operators defined on an arbitrary Fréchet space, we now consider the problem of developing a similar theory within the framework of the dual space $X^{\prime}$. The method we adopt involves the use of adjoint operators, where the latter are defined as in [12]. Bearing this in mind, we concentrate on the following class of operators.

Definition 5.1. The operator $A^{\prime}$ is said to belong to the set $\Pi\left(X^{\prime}\right)$ if $A^{\prime}$ is the adjoint of an operator $A \in P(X)$.

In the sequel, when we refer to an operator $A^{\prime} \in \Pi\left(X^{\prime}\right)$ it is understood that $A^{\prime}$ is the adjoint of the operator $A \in P(X)$. Clearly, any operator $A^{\prime} \in \Pi\left(X^{\prime}\right)$, being the adjoint of a continuous linear operator, is an element in $L\left(X^{\prime}\right)$ [12, p. 29].

Definition 5.2. Let $A^{\prime} \in \Pi\left(X^{\prime}\right)$ and let $\operatorname{Re} \alpha>0$. Then we define the fractional power $\left(-A^{\prime}\right)^{\alpha}$ by 


$$
\begin{aligned}
\left(-A^{\prime}\right)^{\alpha} f= & -\pi^{-1} \sin (\pi \alpha) \int_{0}^{\infty} \lambda^{\alpha-1}\left[R\left(\lambda ; A^{\prime}\right)-\lambda /\left(1+\lambda^{2}\right)\right] A^{\prime} f d \lambda \\
& -\sin (\pi \alpha / 2) A^{\prime} f \quad(0<\operatorname{Re} \alpha<2)
\end{aligned}
$$

and

$$
\left(-A^{\prime}\right)^{\alpha} f=\left(-A^{\prime}\right)^{\alpha-n}\left(-A^{\prime}\right)^{n} f \quad(n<\operatorname{Re} \alpha<n+2, n=1,2, \ldots)
$$

where $f \in X^{\prime}$ and the integral in (5.1) is interpreted as an improper Riemann integral of the form (4.5) convergent in the weak* topology of $X^{\prime}[9, \mathrm{p} .66]$.

That this definition is meaningful is discussed in the proof of the following theorem.

Theorem 5.3. If $A^{\prime} \in \Pi\left(X^{\prime}\right)$, then $\left(-A^{\prime}\right)^{\alpha}$, defined by (5.1) and (5.2), is a continuous linear operator on $X^{\prime}$. Moreover, $\left(-A^{\prime}\right)^{\alpha}$ is the adjoint of $(-A)^{\alpha}$, where $(-A)^{\alpha}$ is defined by (4.3) and (4.4).

Proof. First we remark that $R\left(\lambda ; A^{\prime}\right)=[R(\lambda ; A)]^{\prime}$ exists for any $\lambda>0$ since $A \in P(X)$. Therefore the integrand in (5.1) is well-defined. Now let $\left[\varepsilon_{n}, M_{n}\right]$ be a compact interval of $(0, \infty)$ and let the operator $B_{n, \alpha}$ be defined on $X^{\prime}$ by

$$
B_{n, \alpha} f=\int_{\varepsilon_{n}}^{M_{n}} \lambda^{\alpha-1}\left[R\left(\lambda ; A^{\prime}\right)-\lambda /\left(\lambda^{2}+1\right)\right]\left(-A^{\prime} f\right) d \lambda \quad(0<\operatorname{Re} \alpha<2) .
$$

It can be shown [5, pp. 141-142] that $B_{n, \alpha}$ is the adjoint of the operator $A_{n, \alpha} \in L(X)$ given by

$$
A_{n, \alpha} x=\int_{\varepsilon_{n}}^{M_{n}} \lambda^{\alpha-1}\left[R(\lambda ; A)-\lambda /\left(1+\lambda^{2}\right)\right](-A x) d \lambda \quad(x \in X) .
$$

Therefore, it follows that $B_{n, x} \in L\left(X^{\prime}\right)$. Furthermore, if $\left\{\varepsilon_{n}\right\}$ and $\left\{M_{n}\right\}$ are chosen so that $\varepsilon_{n} \rightarrow 0+$ and $M_{n} \rightarrow \infty$ as $n \rightarrow \infty$, then, for any $f \in X^{\prime}$,

$$
\begin{gathered}
\left|\left(f,\left[A_{n, \alpha}-A_{m, \alpha}\right] x\right)\right| \\
\leqq M_{f} \max _{1 \leqq i \leqq l}\left\{\gamma_{f(i)}\left(A_{n, \alpha} x-A_{m, \alpha} x\right)\right\}
\end{gathered}
$$

where $M_{f}$ is a constant and $\gamma_{f(i)} \in S(i=1, \ldots, l)$ (by [12, Theorem 1.8-1]). Since $\left\{A_{n, a}\right\}$ is a Cauchy sequence in $L_{s}(X)$, the right-hand side of (5.3) converges to 0 as $n, m \rightarrow \infty$. Hence, for each $f \in X^{\prime},\left\{B_{n, \alpha} f\right\}$ is a Cauchy sequence in $X^{\prime}$, where $X^{\prime}$ is equipped with the weak* topology, and therefore, by completeness,

$$
\begin{aligned}
B_{\alpha} f & =\lim _{n \rightarrow \infty} B_{n, \alpha} f \\
& =\int_{0}^{\infty} \lambda^{\alpha-1}\left[R\left(\lambda ; A^{\prime}\right)-\lambda /\left(1+\lambda^{2}\right)\right]\left(-A^{\prime} f\right) d \lambda
\end{aligned}
$$


exists in $X^{\prime}$. In addition, the operator $B_{\alpha}$ defined on $X^{\prime}$ by (5.4), being the adjoint of the operator $T(\alpha) \in L(X)$ given by (4.9), is an element in $L\left(X^{\prime}\right)$. From this, we can conclude that $\left(-A^{\prime}\right)^{\alpha}$ is the adjoint of $(-A)^{\alpha}$ for $0<\operatorname{Re} \alpha<2$ and the general result can now be deducted readily from the definitions of $(-A)^{\alpha}$ and $\left(-A^{\prime}\right)^{\alpha}$ for $n<\operatorname{Re} \alpha<n+2, n=1,2, \ldots$.

From standard results on adjoint operators, it is an easy matter to deduce the following facts.

Theorem 5.4. If $A^{\prime} \in \Pi\left(X^{\prime}\right)$ and $\left(-A^{\prime}\right)^{\alpha}$ is defined by (5.1) and (5.2), then

(i) $\left(-A^{\prime}\right)^{\alpha}\left(-A^{\prime}\right)^{\beta}=\left(-A^{\prime}\right)^{\alpha+\beta}, \operatorname{Re} \alpha, \operatorname{Re} \beta>0$;

(ii) $\left[\left(-A^{\prime}\right)^{\alpha}\right]^{\beta}=\left(-A^{\prime}\right)^{\alpha \beta}, 0<\alpha<1, \operatorname{Re} \beta>0$;

(iii) for each $f \in X^{\prime},\left(-A^{\prime}\right)^{\alpha} f$ is a (weak*) holomorphic $X^{\prime}$-valued function of $\alpha$ in the region $\operatorname{Re} \alpha>0$.

Proof. As an illustration of the techniques involved in proving these results, we shall verify (ii). By Theorem 5.3, $\left(-A^{\prime}\right)^{\alpha \beta}$ exists in $L\left(X^{\prime}\right)$ and is the adjoint of $(-A)^{\alpha \beta}$. Hence, for any $x \in X$ and $f \in X^{\prime}$ we have

$$
\begin{aligned}
\left(\left(-A^{\prime}\right)^{\alpha \beta} f, x\right) & =\left(f,(-A)^{\alpha \beta} x\right) \\
& =\left(f,\left[(-A)^{\alpha}\right]^{\beta} x\right) \quad \text { (by Theorem 4.10(ii)) } \\
& =\left(\left[\left(-A^{\prime}\right)^{\alpha}\right]^{\beta} f, x\right) \quad \text { (by Theorem 5.3) }
\end{aligned}
$$

and this establishes the result.

Suppose now that $A$, in addition to being an element in $P(X)$, possesses an inverse $A^{-1} \in L(X)$. Then $\left(A^{\prime}\right)^{-1}$ exists and we can define $\left(-A^{\prime}\right)^{\alpha}$ for $\operatorname{Re} \alpha \leqq 0$ by

$$
\left(-A^{\prime}\right)^{\alpha} f=\left(-A^{\prime}\right)^{\alpha+n}\left[\left(-A^{\prime}\right)^{-1}\right]^{n} f=\left(-A^{\prime}\right)^{\alpha+n}\left[\left(-A^{-1}\right)^{\prime}\right]^{n} f
$$

where $f \in X^{\prime}$ and the non-negative integer $n$ is such that $\operatorname{Re} \alpha+n>0$. The family of operators $\left\{\left(-A^{\prime}\right)^{\alpha} ; \alpha \in \mathbb{C}\right\}$ can be shown to have the following properties.

Theorem 5.5. Let $A \in P(X)$ possess an inverse $A^{-1} \in L(X)$ and let $\left(-A^{\prime}\right)^{\alpha}$ be defined by (5.1) and (5.2) for $\operatorname{Re} \alpha>0$ and extended via (5.5) to all complex values of $\alpha$. Then the operators $\left(-A^{\prime}\right)^{\alpha}$ satisfy (4.19) and (i), (iii) and (iv) of Theorem 4.11 with $A$ and $X$ replaced by $A^{\prime}$ and $X^{\prime}$ respectively. Moreover, for each $f \in X^{\prime},\left(-A^{\prime}\right)^{a} f$ is a (weak $\left.{ }^{*}\right)$ entire $X^{\prime}$-function of $\alpha$.

Proof. If we notice that, for any complex value of $\alpha,\left(-A^{\prime}\right)^{\alpha}$ is the adjoint of $(-A)^{\alpha}$, then this theorem may be verified by using the corresponding properties of $(-A)^{\alpha}$.

\section{Concluding remarks}

The theory presented here provides the framework for studying fractional powers of particular differential and integral operators defined on some specified Fréchet space $X$. 
More importantly, when $X$ is a space of testing functions [12, p. 39] and the operator $A$ under investigation permits an extension $\tilde{A}$ to the generalised function space $X^{\prime}$ via the adjoint method described in [7, p. 7], then Definition 5.2 forms the basis for constructing fractional powers of the extended operator $-\tilde{A}$. In essence, the problem of obtaining a group of operators $(-\tilde{A})^{\alpha}(\alpha \in \mathbb{C})$ reduces to one of finding a space of testing functions $X$ which is such that the formal adjoint $A^{*}$ is invertible on $X$ and also belongs to $P(X)$. Clearly, the conditions imposed on $A^{*}$ will mean that the space $X$ (if it exists) will be restricted in size. However, since our aim is to define fractional powers of the extended operator $-\tilde{A}$ on $X^{\prime}$, in which a large number of classical functions can usually be identified with regular generalised functions, this presents no limitations to the application of the theory.

Acknowledgement. The contents of this paper form part of the author's Ph.D. thesis written at the University of Strathclyde under the supervision of Dr. A. C. McBride. The author would like to thank Dr. McBride for his advice and encouragement, and also the referee of this paper for the useful suggestions he made.

\section{REFERENCES}

1. A. V. Balakrishnan, Fractional powers of closed operators and semigroups generated by them, Pacific J. Math. 10 (1960), 419-437.

2. A. ERDÉLyi et al., Tables of integral transforms (vol. 2) (McGraw-Hill, New York, 1954).

3. H. W. Hövel and U. WestPhal, Fractional powers of closed operators, Studia Math. 42 (1972), 177-194.

4. H. Komatsu, Fractional powers of operators, Pacific J. Math. 19 (1966), 285-346.

5. W. LAMB, Fractional powers of operators on Fréchet spaces with applications (Strathclyde Univ. Ph.D. Thesis, 1980).

6. A. C. MCBRIDE, A theory of fractional integration for generalised functions (Edinburgh Univ. Ph.D. Thesis, 1971).

7. A. C. McBride, Fractional calculus and integral transforms of generalized functions (Research Notes in Mathematics 31, Pitman, London 1979).

8. G. O. Окікіоци, Aspects of the theory of bounded integral operators in $L^{P}$-spaces (Academic Press, London, 1971).

9. W. Rudin, Functional analysis (Tata McGraw-Hill, New Delhi, 1974).

10. J. Watanabe, On some properties of fractional powers of linear operators, Proc. Japan Acad. 37 (1961), 273-275.

11. K. Yosida, Functional analysis (3rd. ed.) (Springer, Berlin, 1971).

12. A. H. Zemanian, Generalized integral transformations (Interscience, New York, 1968).

Department of Mathematics

UNIVERSITY OF STRATHCLYDE

GlasGow 